\title{
A Medical Image Fusion Algorithm Based on Nonsubsampled Contourlet Transform and Feature Matching
}

\author{
Jionghui Jiang ${ }^{1, a}$, Hui Huang ${ }^{2, b}$, Gang Liu $^{3, c}$ \\ ${ }^{1}$ Zhijiang College of Zhejiang University of Technology, Hangzhou, 310024, China \\ ${ }^{2}$ College of Physics and Electronic Information Engineering, Wenzhou University, 325035, China \\ ${ }^{3}$ Zhejiang University of Technology, Hangzhou, 310024, China \\ ajiangjionghui@zjc.zjut.edu.cn,bhuanghui@wzu.edu.cn,cliugang@zjut.edu.cn
}

Keywords: Image Fusion; NSCT; Directional characteristic

\begin{abstract}
By analysis the regional characteristics of Low-frequency subbands and high-frequency subbands in nonsubsampled Contourlet transform (NSCT) for medical images, we proposed an NSCT-based image fusion algorithm for medical images. For each coefficient of the low-frequency subbands, the regional correlation was considered and the fusion strategy based on regional clarity matching was used; for each coefficient of the high-frequency subbands, the directional characteristics of the subbands were considered and the fusion strategy based on local energy of high-frequency regions was used; after that, the high-frequency fusion coefficient was determined. The proposed algorithm was verified through a simulation experiment on CT, PET and MRI images. The fusion effect was assessed using subjective and objective evaluation indicators. The experiment showed that the proposed algorithm achieved a better visual quality and quantitative indicators for medical images.
\end{abstract}

\section{Introduction}

Fusion of medical images is to integrate the images collected by the same or different techniques on the same target object into one image by extracting and processing the useful information from each image using the image fusion algorithms. Several medical imaging techniques are now in used based on the combination of computer and medical sciences, such as computed tomography (CT), magnetic resonance imaging (MRI) and PET scan. Through the fusion of medical images, several images collected by different techniques are synthesized into a single target image, where the information on bones, soft tissue information as well as their physiological functions is well preserved.

Wavelet transform (WT) has found extensive applications in the fusion of medical images [1][2]. Ranchin T. and Wald L.[3] applied the discrete WT technique to multi-source image fusion in 1993. WT technique has the advantages of excellent time-frequency analysis performance, directional anisotropy and relative independence of different scales. Therefore, WT technique can achieve a better fusion effect than Laplacian Pyramid transform [4]. However, WT involves complex procedures and takes a lot time and storage space. Pennec and Mallat[5] proposed Bandelet transform, and Candes[6] proposed curvelet transform. In 2002, Contourlet transform was presented by M.N.DO and Vetterli M.[7], and wavelet-based Contourlet transform (WBCT) was proposed by Eslami R. and Radha H.[8] in 2004. Nonsubsampled Contourlet transform (NSCT) was put forward by Cunha and Zhou J.P. et al. [9] in 2006. Multi-scale geometric analysis is to achieve an optimal approximation of high-dimensional functions based on its multi-resolution feature and anisotropy. It is more applicable to the representation of sparse 2D image signals than WT.

Utilizing the multi-scale, multi-directional, anisotropy and translation invariance features of NSCT, we proposed a medical image fusion method which integrates NSCT and regional feature matching algorithm. NSCT was first done to the source images to obtain low-frequency and high- 
frequency coefficients under different scales. Regional characteristics of low-frequency and highfrequency subbands in the spatial domain were extracted. Correction factors were assigned to the regional characteristics based on the salient features of different images, and the weight coefficients were determined. A simulation experiment on the fusion of CT 、 PET and MRI images indicated that our algorithm achieves higher fusion performance and better visual effect than WT and Contourlet transform.

\section{NSCT procedures}

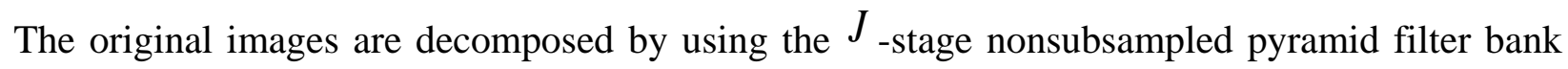
(NSPFB) to obtain low-frequency subband ${ }^{C}$ and high-frequency subband $b_{i}(i=1,2, \ldots, J)$ under different scales. The high-frequency subband $b_{i}$ is subjected to directional decomposition using the nonsubsampled directional filter banks (NSDFB) to obtain the nonsubsampled Contourlet coefficient of directional subbands under a specific scale. This is the basic principle of multi-scale, multi-directional decomposition of the image using NSCT. For each scale, decomposition leads to $2^{l}$ directional subbands; for every two scales, the number of directional subbands will increase by one-fold, which makes anisotropy and sparse image representation possible.

\subsection{NSPFB}

NSPFB mainly consists of an iterative process through a two-channel filter bank. Since no subsampling is included, translation invariance is well preserved. One bandpass image is generated at each stage of NSPFB, as shown in Fig. 1.

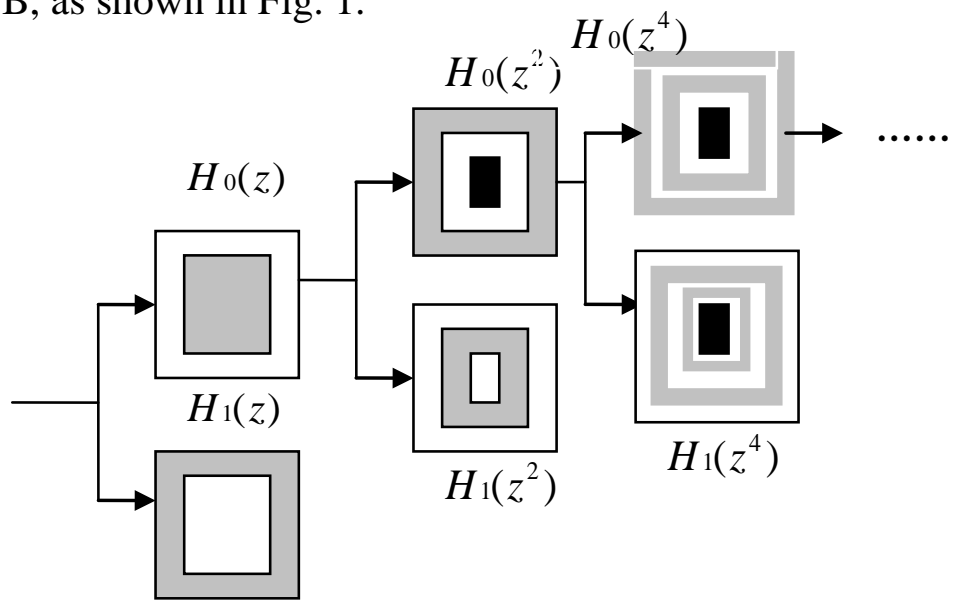

Fig. 1 Schematic of NSPFB

If $H_{0}(z)$ and $H_{1}(z)$ satisfy the conditions of perfect reconstruction, there is no need for designing additional filters. Filters of the next stage can be obtained by upsamping the filters of the previous stage with sampling matrix $D=2 I$. That is, NSFB can be used iteratively to construct NSPFB for multi-scale decomposition. Filters generated in this way still satisfy the conditions of perfect reconstruction. The nonsubsampled pyramid filter of the $n$-th stage is represented by

$$
H_{n}(z)=\left\{\begin{array}{lc}
H_{1}(z) \prod_{j=0}^{n-2} H_{0}\left(z^{2^{j}}\right) & 1 \leq n \leq 2^{k} \\
\prod_{j=0}^{n-1} H_{0}\left(z^{2^{j}}\right) & n=2^{k}
\end{array}\right.
$$

\subsection{Nonsubsampled directional filter bank (NSDFB)}

However, translation invariance is broken with conventional Contourlet transform because of uniform sampling of the translation parameters. Here the NSDFB replaces DFB (critical sampling) to perform directional decomposition of the frequency spectra using the two-channel fan filter bank 
(precise sampling) and resampling. Fig. 2 shows the schematic of the iteration of two-channel fan filter bank to produce a one-channel NSDFB.

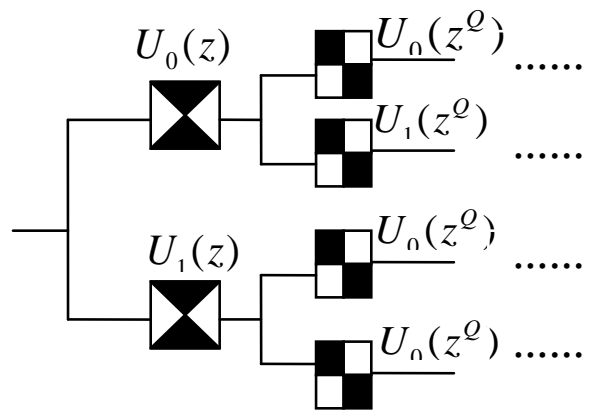

Fig. 2 Schematic of NSDFB

$\left\{U_{0}(z), U_{1}(z)\right\}$ represents complementary fan filter banks and iteration of NSDFB is performed. Quadrant filter is $U_{i}\left(z^{Q}\right), i=0,1, Q=\left(\begin{array}{rr}1 & 1 \\ 1 & -1\end{array}\right)$. Subbands obtained by decomposition on the previous stage are filtered to obtain directional subbands with the same size of the source image. Equivalent filter in each channel satisfies

$$
U_{k}^{e q}(z)=U_{i}(z) U_{j}(z)
$$

\section{Feature matching algorithm}

\subsection{Fusion strategy for low-frequency subbands}

Low-frequency subbands obtained by NSCT are approximate subbands of the source images and they reflect the general information of the source images. The selected regional characteristics must be normalized, and assessment of the structural similarity between the two images is needed. The commonly used evaluation indicators are regional information entropy $S$, regional energy $E$, maximum and minimum of regional gray scale, and regional mean or median. Based on the regional characteristics of the transform coefficient, clarity is taken as the assessment criterion. Clarity at pixel $(x, y)$ on the image is defined as the vector norm sum of image gray gradient within the window ${ }^{w}(3 \times 3$ or $5 \times 5$ pixel $)$ which takes the studied pixel as the center:

$$
\beta=\sum_{(x, y) \in w}\left\{[f(x, y)-f(x+1, y)]^{2}+[f(x, y)-f(x, y+1)]^{2}\right\}
$$

$\beta$ is the gray scale transform rate within the window; the smaller the value, the more obscure the image is, and vice versa. The assessment criterion used for the fusion is represented by

$$
C_{k}= \begin{cases}C_{a k}(x, y) & \beta_{a k}>\beta_{b k} \\ C_{k b}(x, y) & \beta_{a k} \leq \beta_{b k}\end{cases}
$$

\subsection{Feature matching strategy for high-frequency subbands}

The feature matching strategy is used for the fusion (Salience/Match Measure with Threshold). The feature matching strategy for local high-frequency energy is defined as follows [10]:

$$
S(i, j, k)=\sum_{m} \sum_{n} C(i+m, j+n, k)^{2}
$$

where $C(i, j, k)$ is high-frequency coefficient for $(i, j)$ at the $k$ the decomposition stage; $(m, n)$ is the size of a neighborhood window on the image $(3 \times 3$ or $5 \times 5$ pixel). The sum of squares of highfrequency coefficients in this neighborhood is the local energy at $(i, j, k)$; the matching degree for $(i, j, k)$ is defined as 


$$
M_{A B}(i, j, k)=\frac{2 \sum_{m} \sum_{n} C_{A}(i+m . j+n, k) C_{B}(i+m . j+n, k)}{S_{A}(i, j . k) S_{B}(i, j . k)}
$$

The fusion strategy for high-frequency coefficient is defined by

$$
C(i, j, k)=W_{A} C_{A}(i, j, k)+W_{B} C_{B}(i, j, k)
$$

That is, the high-frequency coefficient after fusion is the weighted sum of the coefficients of the original images at this position. The weight is determined using the following method:

1. If $M_{A B} \leq T$, then

$$
\begin{cases}W_{A}=1, W_{B}=0 & S_{A}>S_{B} \\ W_{A}=0, W_{B}=1 & S_{A} \leq S_{B}\end{cases}
$$

2. If $M_{A B}>T$, then

$$
\begin{cases}W_{A}=1-W_{B}, W_{B}=1 / 2-\left(1-M_{A B}\right) / 2(1-T) & S_{A}>S_{B} \\ W_{A}=1 / 2-\left(1-M_{A B}\right) / 2(1-T), W_{B}=1-W_{A} & S_{A} \leq S_{B}\end{cases}
$$

Where $T \in(0,1)$ is the threshold of matching degree. When matching degree $M_{A B}$ is less than the threshold $T$, choose the larger values of local energy $C_{A}(i, j, k)$ and $C_{B}(i, j, k)$. When matching degree $M_{A B}$ is larger than the threshold $T$,high-frequency fusion coefficient is $W_{A} C_{A}(i, j, k)+W_{B} C_{B}(i, j, k)$.The weights of $W_{A}$ and $W_{B}$ are related to the matching degree $M_{A B}$, and $W_{A}+W_{B}=1$. Obviously, the calculation process of the feature matching rules has good local properties. The high-frequency coefficient for $(i, j, k)$ after fusion is only determined by the coefficients in $\mathrm{m} \times \mathrm{n}$ neighborhood for $(i, j, k)$.

\section{Experiment and analysis}

Image fusion was performed in Matlab7.0 simulation and the result was compared with Wavelet weighted local contrast fusion(Wavelet Fusion)[11], weighted Contourlet transformation coefficients fusion(Contourlet Fusion)[12] and nonsub sampled contourlet transform Fusion(NSCT Fusion)[13].The region used for the fusion was 3x3 in size. The number of decomposition stages was 3 for the Contourlet transform. The threshold of matching degree for the high-frequency subbands was set to 0.75 . The weight factor of WT was 0.5 .

Fig. 3(a) and (b) and Fig. 4 (a) and (b) are the two groups of calibrated medical source images, respectively. Fig. 3 (c) and Fig. 4(c) are the results of Wavelet weighted local contrast fusion(Wavelet Fusion). Fig. 3(d) and Fig. 4 (d) are the results of weighted Contourlet transformation coefficients fusion(Contourlet Fusion). Fig. 3(e) and Fig. 4(e) are the results of nonsubsampled contourlet transform Fusion(NSCT Fusion).Fig. 3(f) and Fig. 4(f) are the results of the proposed algorithm. As to the visual effect, the fused image has a light color using Wavelet Fusion, and the edge contour is not distinct; the fused image fails to reflect the details on the medical source images. The fusion effect with the Contourlet Fusion is slightly better as compared with Wavelet Fusion, and the former can more clearly display the information from the source images. NSCT Fusion and proposed algorithm is well preserved in medical images of bone and soft tissue soft tissue, which the fusion image edge details highlight, and better than Wavelet Fusion and Contourlet Fusion. Based on the qualitative analysis of the experimental results of Fig.3 and Fig. 4, a quantitative evaluation was further conducted using objective indicators, namely, standard deviation, Spatial frequency, entropy and mutual information. The evaluation indicators can reflect the quality of the fused image[14]. 


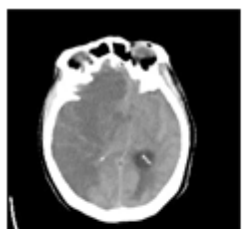

(a) CT Image

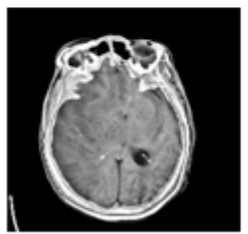

(d) Contourlet Fuion

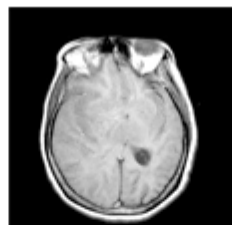

(b) MRI Image

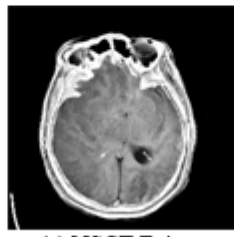

(e) NSCT Fuion

In Literature[16]

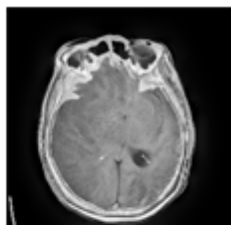

(c) Wavelet Fusion

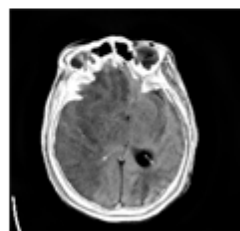

(f) Proposed Algorithm

Fig. 3 Fusion result of CT and MRI images

Table 1 Evaluation indicators for CT and MRI images

\begin{tabular}{ccccc}
\hline & Standard deviation & Spatial frequency & Entropy & Mutual information \\
Wavelet Fusion [14] & 43.867 & 5.277 & 4.058 & 1.208 \\
Contourlet Fusion [15] & 45.384 & 6.718 & 6.047 & 2.509 \\
NSCT Fusion [16] & 50.720 & 6.832 & 6.246 & 2.559 \\
Proposed Algorithm & 52.164 & 6.841 & 6.344 & 2.641 \\
\hline
\end{tabular}

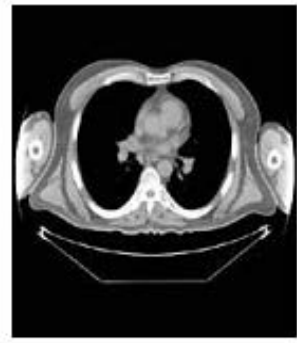

(a) CT Image

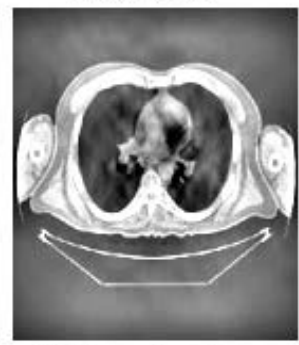

(d) Contourlet Fuison

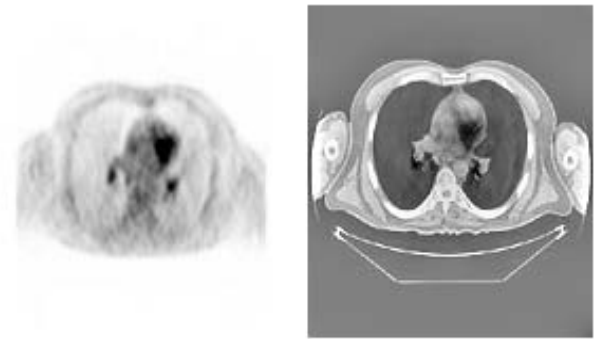

(b) PET Image

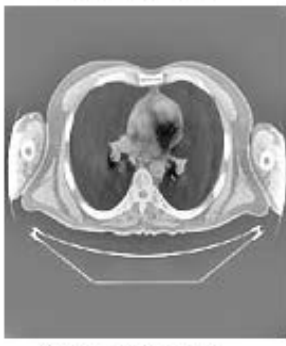

(e) NSCT Fusion In Literature[16] (c) Wavelet Fusion

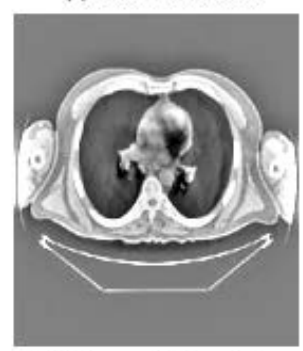

(f) Proposed Algorithm

Fig. 4 Fusion result of CT and PET images

Table 2 Evaluation indicators for CT and PET images

\begin{tabular}{ccccc}
\hline & $\begin{array}{c}\text { Standard } \\
\text { deviation }\end{array}$ & Spatial frequency & Entropy & Mutual information \\
\hline Wavelet Fusion [14] & 76.270 & 8.492 & 2.242 & 2.216 \\
Contourlet Fusion [15] & 81.304 & 8.801 & 4.391 & 3.266 \\
NSCT Fusion [16] & 81.483 & 9.463 & 5.233 & 3.321 \\
Proposed Algorithm & 83.154 & 9.621 & 5.311 & 3.354 \\
\hline
\end{tabular}

Table 1 and table 2 give a variety of objective evaluation indicators of the experimental results of Fig3 and Fig 4.Table 1 and 2 show the objective indicator values of the fused images obtained by different methods. The standard deviation, Spatial frequency, entropy and mutual information with Wavelet Fusion are the smallest, indicating more considerable loss of information during fusion and 
greater distortion of the source images. Thus Wavelet Fusion has a poor fusion performance. Contourlet Fusion leads to more directional high-frequency subbands than Wavelet Fusion, so the former is better in processing the directional details and achieves a more satisfactory visual effect than the latter. NSCT Fusion's index coefficients better than Wavelet Fusion and Contourlet Fusion, but in the high-frequency sub band fusion algorithm is not accurate enough, resulting in the overall fusion quality is not very high。 The indicator values are the highest with the proposed algorithm, indicating a good similarity of the fused image with the source images. Some important details are preserved from both CT , PET and MRI source images. Therefore, the propose algorithm is the best and achieves the most excellent visual effect.

Figure 5 and Figure 6 are the other two groups of medical images using the results of different fusion algorithms. Table 3 and table 4 are the objective evaluation of the experiment. From the subjective visual and objective evaluation index of image experiment results, proposed Algorithm is also better than Wavelet weighted local contrast fusion(Wavelet Fusion), weighted Contourlet transformation coefficients fusion(Contourlet Fusion) and nonsubsampled contourlet transform Fusion(NSCT Fusion).

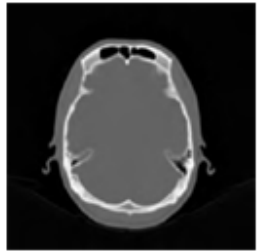

(a) CT Image

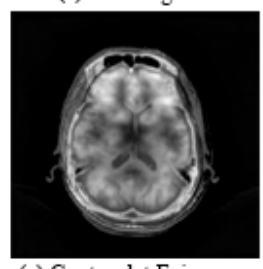

(e) Contourlet Fuion

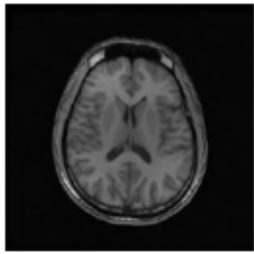

(b) MRI Image

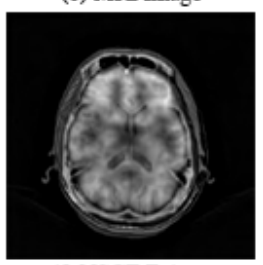

(f) NSCT Fuion

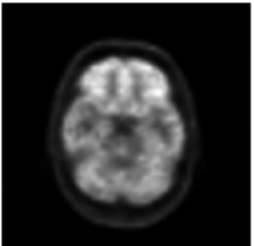

(c) PET Image

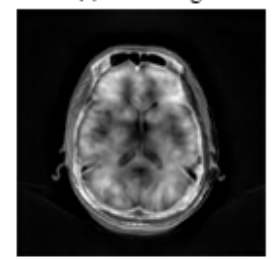

(g) Proposed Algorithm

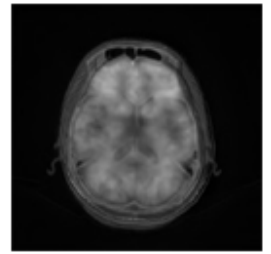

(d) Wavelet Fusion

Fig. 5 Fusion of CT, MRI and PET images

Table 3 Evaluation indicators of Fig.5

\begin{tabular}{ccccc}
\hline & Standard deviation & Spatial frequency & Entropy & Mutual information \\
\hline Wavelet Fusion [14] & 47.278 & 16.254 & 5.025 & 1.823 \\
Contourlet Fusion & 53.472 & 18.349 & 5.188 & 2.541 \\
[15] & & & & \\
NSCT Fusion [16] & 59.526 & 19.340 & 5.245 & 2.642 \\
Proposed Algorithm & 61.404 & 19.436 & 5.266 & 2.710 \\
\hline
\end{tabular}

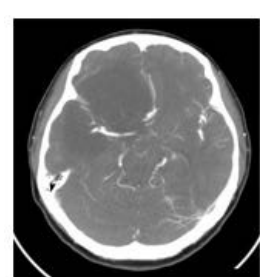

(a) CT Image

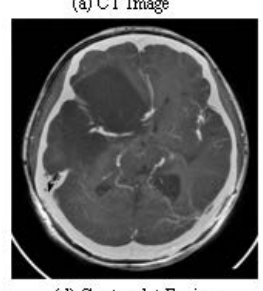

(d) Contourlet Fusion

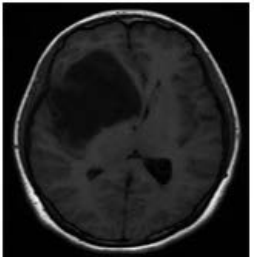

(b) MRU Image

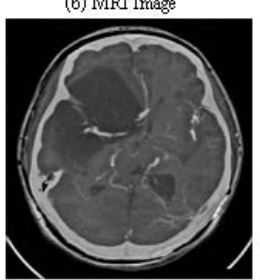

(e) NSCT Fuion In Literature[16]

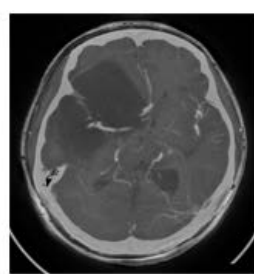

(c) Wavelet Fusion

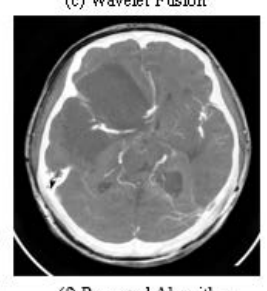

(f) Proposed Algorithm

MRI 
Table 4 Evaluation indicators of Fig.6

\begin{tabular}{ccccc}
\hline & Standard deviation & Spatial frequency & Entropy & Mutual information \\
\hline Wavelet Fusion [14] & 48.351 & 15.318 & 5.883 & 2.531 \\
Contourlet Fusion & 53.355 & 17.380 & 6.157 & 3.264 \\
[15] & & & & \\
NSCT Fusion [16] & 64.629 & 17.668 & 6.181 & 3.434 \\
Proposed Algorithm & 65.181 & 17.980 & 6.461 & 3.556 \\
\hline
\end{tabular}

\section{Conclusions}

We combined NSCT with regional feature matching algorithm for the low-frequency and highfrequency subbands and proposed a new method for medical image fusion. As compared with Wavelet weighted local contrast fusion(Wavelet Fusion), weighted Contourlet transformation coefficients fusion(Contourlet Fusion) and nonsubsampled contourlet transform Fusion(NSCT Fusion), the proposed algorithm can better integrate the details from CT, PET and MRI source images and achieve a better visual effect. NSCT has a good locality in spatial and frequency domains, which enables the separation of low-frequency and high-frequency subbands. Therefore, the fusion can be done separately in different channels. Fusion experiment on CT, PET and MRI source medical images demonstrates that the proposed algorithm outperforms the other two algorithms in both visual effect and objective evaluation indicators.

\section{Acknowledgements}

This work is supported by the Zhejiang Provincial Natural Science Foundation ofChina (Grant No. LY15F020033), andthe Science and TechnologyPlan Project of Wenzhou, China (Grant No. Y20160070).

\section{References}

[1] Yang LC, Liu YM, Liu X, Yao YC. A medical image fusion algorithm based on wavelet transform [J]. Chinese Journal of Biomedical Engineering, 2009:28(1):12-16.

[2] Li JF, Jiang XL, Dai WZ. Medical image fusion based on lifting wavelet transform [J]. Journal of Image and Graphics, 2014:19(11):1639-1645.

[3] Ranchin T, Wald L. The wavelet transform for the analysis of remotely sensed images [J]. International Journal of Remote Sensing, 1993, 14(3) : 615-619.

[4] Burr P J, Adelson E H. The laplacian pyramid as a compact image code [J]. IEEE Transactions on Communications, 1983, 31 (4):532-540.

[5]Pennec,Mallat.Sparsegeo metric image representation sbandelets[J].IEEE Transactions Image Processing,2005,14(4):423-438

[6] Candes Emmanuel, DEMANET Laurent,DONOHO David, et al. Fast discrete Curvelet transforms [J]. Society for Industrial and Applied Mathematics, 2006, 5 (3) : 861-899.

[7] Do MN, Vetterli M. The contourlet transform: an efficient directional multiresolution image representation[J]. IEEE Transactions on image Processing. 2005, 14(12): 2091-2106.

[8] R. Eslami and H. Radha. "Wavelet-based contourlet transform and its application to image coding in proc. Of IEEE International Conference on Image Processing, Oct. 2004.

[9] Cunha AL, Zhou Jiangping, Do MN. The nonsubsampledcontourlet transform: theory,design,and applications[J]. IEEE Transactions on image Processing. 2005, 15(10): 30893101. 
[10] Xue XC, Zhang SY, Li HF, Guo YF. A study on the application of Contourlet transform in image compression [J]. Microcomputer Information, 2009 (25).

[11]Yang,Yan-chun,Wang Xiao-mingetc.Medical image fusion method based on wavelet weighted local contrast[J]. Journal of Lanzhou University(Natural Sciences), 2013,49(1):122-125

[12] Zhang Xin,ChenWeibin. Medical image fusion based on weighted Contourlet transformation coefficients[J]. Journal of Image and Graphics,2014,19(1):133-140.

[13] JIN Zhen-yi,WANG Yuan-jun. Multi-modality medical image fusion method based on nonsubsampled contourlet transform[J]. Chinese Journal of Medical Physics,2016,33(5):445-450

[14] ZHANG Xiao-Li, LI Xiong-Fei1,LIJun.Validation and Correlation Analysis of Metrics forEvaluating Performance of Image Fusion [J]. ACTA AUTOMATICA SINICA. 2014,40(2):306314 